\title{
ESTACIONALIDAD Y ESTRUCTURA DE LA VEGETACIÓN EN LA COMUNIDAD DE ANFIBIOS Y REPTILES DE CHAMELA, JALISCO, MÉXICO
}

\author{
García, A' 1 y A. Cabrera-Reyes ${ }^{2}$ \\ 1Estación de Biología Chamela, Instituto de Biología, Universidad Nacional Autónoma de \\ México, Ap. Postal 21, San Patricio, Melaque, Jalisco, 48980, MEXICO, correo electró- \\ nico: chanoc@ibiologia.unam.mx, teléfono (01-315) 3510202. \\ 2Facultad de Ciencias Biológicas, Universidad Autónoma del Estado de Morelos, Av. \\ Universidad 1001, Col. Chamilpa, Cuernavaca, Morelos, 62209, MEXICO
}

\section{RESUMEN}

Se evaluó el efecto de la estacionalidad ambiental y la estructura de la vegetación en los patrones espacio-temporales de la comunidad (composición, diversidad, riqueza y abundancia relativa) de los anfibios y reptiles de la región de Chamela, Jalisco. Tres sitios fueron muestreados, mediante un diseño de bloque dentro de una matriz de vegetación de arroyo (VA) y bosque tropical caducifolio (BTC) utilizando tres técnicas complementarias de muestreo (trampeo; parcelas de búsqueda intensiva y transectos visuales) durante la época de secas y de lluvias. Se registraron 34 especies (25 de reptiles y nueve de anfibios). Las curvas de acumulación de especies y los índices predictores de riqueza, sugieren que el esfuerzo de muestreo fue el adecuado. Los valores más altos de riqueza, diversidad y abundancia se registraron en la VA. Se registraron mayores fluctuaciones estacionales en anfibios que en reptiles, y en el BTC que en la VA. Se documenta un recambio de especies, tanto espacial (matriz de vegetación VA-BTC) como temporal (estacional), más marcado en anfibios cuyo registro de individuos fue mínimo durante la época de secas y solo dentro de la VA. La VA es importante para el mantenimiento de la diversidad herpetofaunística, no sólo por su menor estacionalidad y mayor productividad, sino por su mayor complejidad estructural. Se encontró una mayor respuesta de los anfibios hacia los cambios en la estructura de la vegetación que los reptiles, los cuales estuvieron más ampliamente distribuidos espacial y temporalmente.

Palabras clave: bosque tropical caducifolio; herpetofauna; estacionalidad ambiental; vegetación; estructura de la comunidad

\begin{abstract}
The effect of effect of environmental seasonality and habitat structure on the spatial and temporal patterns of the reptile and amphibian communities in Chamela, Jalisco, Mexico was evaluated. Three complementary sampling techniques (plots, visual transects and sampling arrays with drift fences and pitfall traps) were used in three sites during the wet and dry seasons using a block design to sample the seasonally dry tropical forest (SDTF) and its adjacent arroyo forest (VA). Thirty-four species were recorded (25 of reptiles and 9 of amphibians). The species accumulation curves and richness predictors suggested sampling effort was appropriated. Highest values of species richness, diversity and relative abundance were recorded in the VA when compared with the SDTF. Seasonal fluctuations were marker in the amphibian community and in the SDTF than in reptiles and the VA respectively. There is a marked species turn over both spatially (along the VA and SDTF) and temporally (across the seasons) more
\end{abstract}


especially evident in amphibians. Arroyo forest is important for the maintenance of species diversity, especially for amphibians, because in this vegetation environmental seasonality is less marked, and both productivity and vegetation structure is higher. The response of amphibians to changes in vegetation structure was more marked than those recorded in reptiles.

Keywords: seasonally tropical dry forest; herpetofauna; environmental seasonality; vegetation; community structure

\section{INTRODUCCIÓN}

La estacionalidad ambiental afecta la estructura de las comunidades ecológicas a través de los cambios temporales en la disponibilidad de los recursos (espacio y alimento), temperatura, agua y fotoperíodo. Las especies animales deben sincronizar aspectos básicos de su biología y ecología como son, la reproducción, el crecimiento, la actividad diaria y anual y la distribución espacial, entre otros, ante las variaciones estacionales. Lo anterior provoca fluctuaciones estacionales en la riqueza, composición, abundancia y diversidad de las comunidades (Lister 1980, Leighton \& Leighton 1983, Wolda 1988, Allmond 1991, Duellman 1995, Duellman \& Thomas 1996). Sin embargo, la importancia de estos factores ambientales difiere entre regiones, siendo las fluctuaciones estacionales en temperatura más importantes en regiones templadas, mientras que las de precipitación lo son en regiones tropicales y áridas (Leighton \& Leighton 1983, Wolda 1988, Potts 2003).

En otro contexto, la complejidad estructural del hábitat, como una forma de heterogeneidad espacial, puede promover una mayor diversidad al ofrecer diversas alternativas de hábitat a las especies, sobre todo de aquellas que tienen preferencias por un tipo de hábitat particular (MacArthur \& MacArthur 1961, Tews et al. 2004). De esta manera, la existencia de zonas de transición o gradientes de vegetación será un promotor de la diversidad sí se registran patrones definidos en la asociación de las especies con los distintos tipos de vegetación presentes en un área o con algunas de sus características en particular (Brown \& Lomolino 1998, Coulson 1993, Williams et al. 1997, Sanders \& Edge 1998).

El bosque tropical caducifolio (BTC) es uno de los principales tipos de vegetación del occidente de México (Trejo-Vázquez 1998, García 2006). Se caracteriza por una prolongada época de secas que puede durar hasta ocho meses, y una corta época de lluvias que por lo general ocurre entre julio y octubre (e.g. Murphy \& Lugo 1986, Bullock et al. 1995; Noguera et al. 2002). En la región de Chamela, en la costa de Jalisco, el paisaje está dominado por pequeños lomeríos cubiertos por BTC y por vegetación de arroyo (VA) que se desarrolla en los pequeños valles adyacentes (Lott et al. 1987, Noguera et al. 2002). Si bien, ambos tipos de vegetación se encuentran en las mismas condiciones climáticas, la topografía permite la acumulación de agua en el lecho de los arroyos donde se desarrolla la VA, por lo que, en ésta hay más especies perenifolias, mayor productividad, complejidad estructural y menor estacionalidad en la disponibilidad de recursos al compararla con el BTC, diferencias 
que son aún más marcadas durante la época de secas (Bullock et al. 1995, Lott et al. 1987, Noguera et al. 2002). De esta manera, ambos tipos de vegetación forman una matriz VA-BTC, la cual muestra marcados contrastes estacionales en su fisonomía, estructura y productividad (Lott et al. 1987, Ceballos 1989, 1990, 1995, MartinezYrízar et al. 1996, García 2003). La estacionalidad ambiental y las características contrastantes de la VA y BTC, representan un excelente escenario para estudiar el efecto de la estacionalidad ambiental y estructura de vegetación sobre la riqueza, diversidad y abundancia de la herpetofauna de la región de Chamela, lo que constituye el objetivo principal de este estudio.

En México, el BTC está fuertemente amenazado por la modificación del hábitat, con una tasa de deforestación anual del 2\% (Trejo-Vazquez y Dirzo 2000), así como por la amenaza del calentamiento global con sus impactos negativos en la estructura de las comunidades y la distribución de sus especies (Villers-Ruiz \& Trejo-Vázquez 1997). Problemas tan graves como la aparente declinación global de las poblaciones de anfibios (Beebee 2002) requieren de la creación de base de datos y marcos de referencia que permitan establecer los alcances de este fenómeno, su caracterización y posible manejo o solución. Sí bien el BTC y sus especies asociadas han comenzado a ser estudiadas en mayor detalle (ej. Bullock 1995, Noguera et al. 2002), existiendo algunos estudios sobre comunidades herpetofaunísticas de este tipo de vegetación en Centro América (e.g. Scott 1976, Fauth et al. 1989, Duellman \& Thomas 1996), en México los estudios de comunidades de reptiles y anfibios del BTC son escasos.

\section{MÉTODOS}

Área de estudio. El estudio se realizó en la Estación de Biología Chamela del Instituto de Biología de la UNAM que forma parte de la Reserva de la Biosfera Chamela-Cuixmala en la costa de Jalisco, Municipio de la Huerta y que protege un poco más de 13000 has de BTC (Ceballos et al. 1999). El acceso a la Estación se ubica en el Km. 59 de la carretera Federal 200, que une Barra de Navidad y Puerto Vallarta (Fig. 1). El paisaje de la región está dominado por pequeños lomeríos cubiertos por el BTC y valles donde se desarrolla la VA (Lott et al. 1987). La información general sobre las características físicas y biológicas del área pueden obtenerse de varias fuentes (e.g. Bullock 1986, Lott et al. 1987, Ceballos et al. 1999, Noguera et al. 2002). La zona de estudio se caracteriza por una marcada estacionalidad en la precipitación, con una corta temporada de lluvias (julio-octubre) y una prolongada sequía. La temperatura media anual es de $24.9^{\circ} \mathrm{C}$ y la precipitación promedio anual de $748 \mathrm{~mm}$. Existen 10 tipos de vegetación, entre los que sobresalen por su cobertura, el BTC, la selva mediana subperenifolia y la VA. La diversidad florística y faunística de la región es considerable, no sólo en términos de riqueza de especies, sino también de endemismos. En la actualidad se tienen registradas aproximadamente 1200 especies de plantas vasculares (Lott et al. 1987, Noguera et 


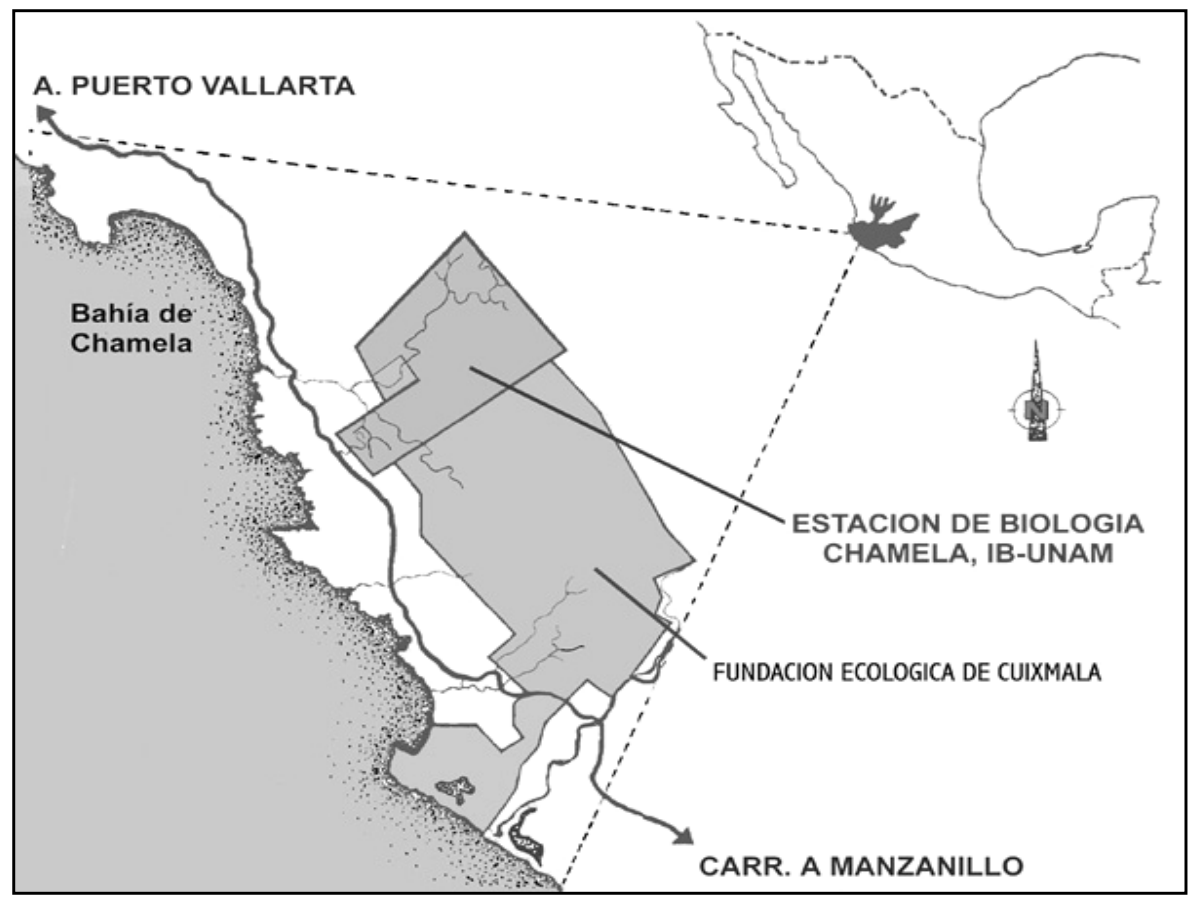

Figura 1. Ubicación del área de estudio.

al. 2002) y 430 de vertebrados con un endemismo del 10 y $19 \%$ respectivamente (Ceballos et al. 1999, Noguera et al. 2002). La herpetofauna de Chamela está compuesta por 87 especies que incluyen 68 de reptiles y 19 de anfibios (García \& Ceballos 1994, Ramírez-Bautista 1994, Ramírez-Bautista \& García 2002, RamirezBautista \& Moreno 2006).

Muestreo de especies. El muestreo se realizó durante los bimestres de abril-mayo y agosto-septiembre del 2004, éstos coinciden con el pico de las épocas de seca y lluvias respectivamente. Se eligieron tres sitios de muestreo (Sitio 1 Búho, Sitio 2 Calandria y Sitio 3 Hornitos), separados por un $\mathrm{km}$ de distancia aproximadamente, todos en zonas de contacto de BTC y VA. En cada sitio de muestreo se trazó una línea de $400 \mathrm{~m}$ de longitud donde se ubicaron cuatro estaciones de muestreo (E1, E2, E3 y E4) espaciadas por $130 \mathrm{~m}$ iniciando desde el centro de la VA (E1) hasta un sitio con BTC siguiendo el gradiente en estructura de vegetación y microclima registrado en el mosaico VA-BTC (Lott et al. 1987). En cada estación de muestreo se utilizaron tres técnicas complementarias de muestreo (ver adelante en el texto). La instalación de estaciones de muestreo (con una, o diferentes técnicas de muestreo) espaciadas regularmente a lo largo de líneas extensas de muestreo (Fig. 2), 
es recomendada en estudios que como éste, investigan los cambios en las comunidades herpetofaunísticas a lo largo de gradientes o a través de ecotonos (e.g. Szaro \& Belfit 1986, Pearman et al. 1995, Hoffer et al. 1999). La longitud de esta línea $(400 \mathrm{~m})$ está dada por la separación que existe entre el centro de la VA y la cima del lomerío adyacente cubierto de BTC. De esta forma, la distribución espacial de los sitios y estaciones de muestreo permitió estudiar la comunidad de reptiles y anfibios dentro de dos tipos de vegetación adyacentes, pero con características contrastantes en su estacionalidad, fisonomía, estructura y productividad.

Las tres técnicas complementarias empleadas para el registro de reptiles y anfibios en cada estación de muestreo (e.g. E1) incluyeron sistemas de trampeo (trampas de caída y cercas de desvío), parcelas de búsqueda intensiva y transectos visuales. Estas son técnicas estandarizadas que han permitido la fácil comparación entre estudios y localidades (e.g. Heyer \& Berven 1973, Heyer et al. 1994, Hofer \& Bersier 2001, Metts et al. 2001, Homyack \& Giuliano 2002, Vallant 2002, Gardner 2007). El transecto visual correspondió a la línea de muestreo trazada para la ubicación de las estaciones de muestreo (de $400 \mathrm{~m}$ de longitud) pero con un ancho de $5 \mathrm{~m}$ (Crump \& Scott 1994, Pearman et al. 1995), mismo que siguió la matriz VA-BTC. El transecto inicio en la parte central de la VA continuándose a través del BTC. De esta manera, en cada sitio de muestreo se utilizaron cuatro sistemas de trampeo, cuatro parcelas y un transecto (Fig. 2). Se requirieron dos semanas para el muestreo de todos los sitios mediante todas las técnicas, en la primera se hizo el trampeo y en la siguiente las parcelas (los tres primeros días) y transectos visuales (los siguientes), realizándose así, durante cada bimestre (ocho semanas), cuatro muestreos por técnica. A continuación se describen brevemente las técnicas de muestreo empleadas en este estudio.

Los sistemas de trampeo consistieron en la combinación de tres cercas metálicas de desvío ( $3 \times 0.7 \mathrm{~m}$ ), enterradas $30 \mathrm{~cm}$ en un arreglo concéntrico y seis trampas de caída (cubetas de 19 L) colocadas a cada extremo de las cercas (Heyer et al. 1994). Las trampas se abrieron y mantuvieron así durante cinco días en las que se revisaron diariamente, iniciando a las 09:00 horas, dejándolas por periodos de $24 \mathrm{hrs}$ (cinco períodos). Los transectos visuales se realizaron en un sitio por día a la misma hora, de 1000-1300 horas, caminando lentamente y recorriéndolo tres veces, en las que se registraron los organismos presentes tomando los siguientes datos: hora de registro, temperatura ambiental, porcentaje de humedad ambiental, tipo de vegetación, fecha y especie. En cada estación de muestreo se trazó un cuadrante de $900 \mathrm{~m}^{2}$ que fue subdividido en 36 parcelas de 5 x 5 m (Fauth 1989, Heinen 1992). En cada muestreo se revisó una parcela que fue elegida al azar de entre las 36 potenciales. Durante la revisión de la parcela, se removió a mano toda la hojarasca y otros materiales caídos, examinándose los árboles en busca de especímenes. 


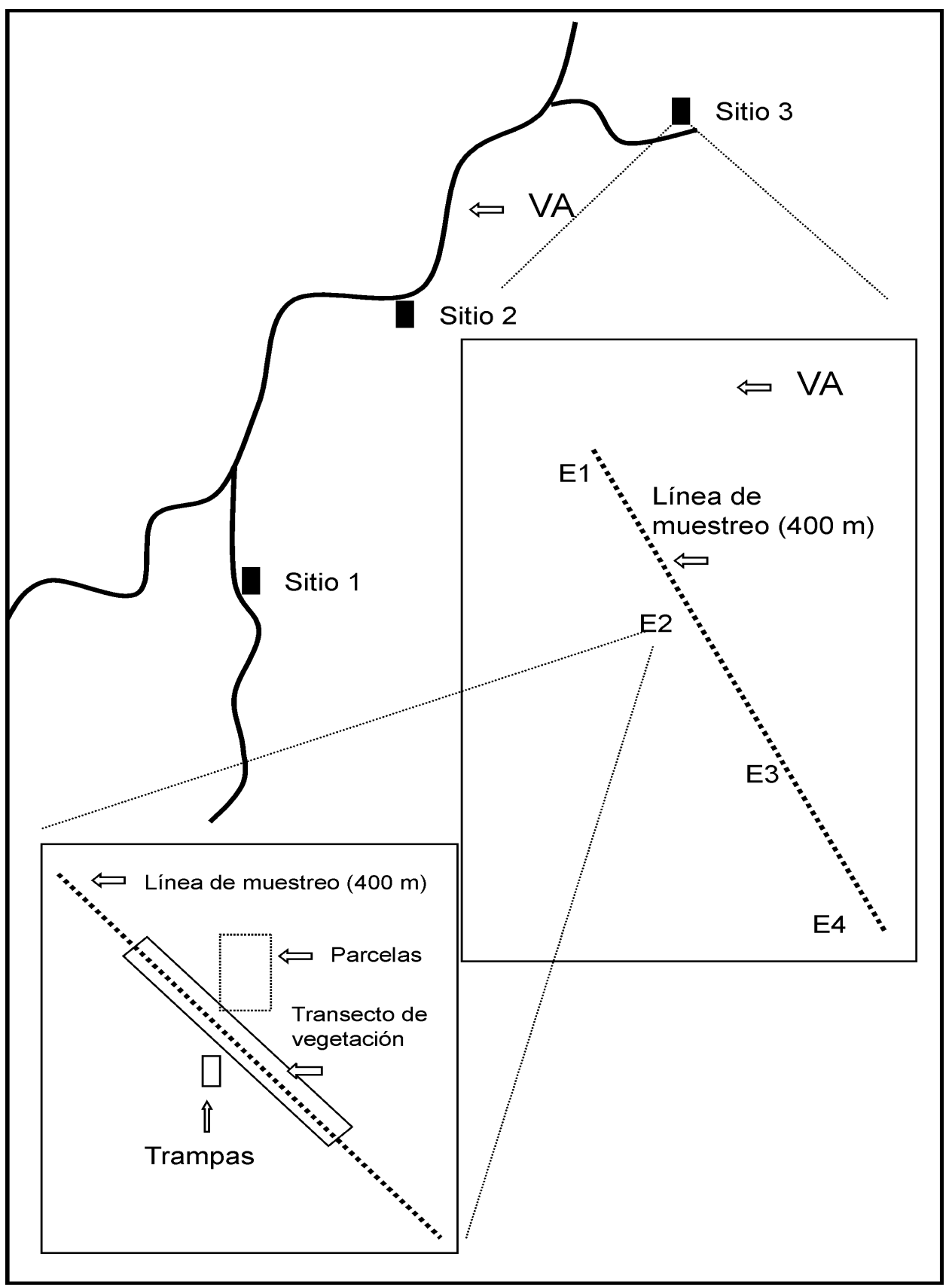

Figura 2. Esquematización de la ubicación de los sitios de muestreo y la distribución de las estaciones y técnicas de muestreo en cada sitio con respecto a la vegetación de arroyo (VA). 
El manejo de los especimenes se realizó en el campo, siendo liberados en el mismo punto de colecta. Cada ejemplar capturado u observado fue identificado hasta el nivel de especie utilizando las guías de campo publicadas de Chamela (García \& Ceballos 1994, Ramírez-Bautista 1994, Ramírez-Bautista \& Moreno 2006). Para cada ejemplar capturado de todas las especies se obtuvieron los datos merísticos estándares y se marcaron por medio de la técnica estándar de ectomización de falanges (Heyer et al. 1994). Las falanges ectomizadas fueron conservadas en alcohol al 70\% para su posterior uso en estudios genéticos, ADN o de otra índole que pudieran surgir en el futuro.

Determinación de la estructura de la vegetación. La medición de la estructura de la vegetación se realizó mediante técnicas estándar siguiendo lo establecido por Lott et al. (1987). Se trazaron cuatro transectos de 2 x $50 \mathrm{~m}$ en cada sitio de muestreo, uno en cada estación de muestreo y siguiendo la línea de muestreo del sitio, fijando el punto medio de estos transectos (el metro 25) justo donde se ubican las trampas y parcelas. En estos transectos se registró, 1) número de árboles con diámetro a la altura del pecho (DAP) $>2.5 \mathrm{~cm} ; 2$ ) número de bejucos y su diámetro a $1.4 \mathrm{~m}$ de la raíz; 3) número de ramas de árboles cuya ramificación iniciaba a $1.5 \mathrm{~m}$ y cuyas ramas midieran más de $2.5 \mathrm{~cm}$. de diámetro; 4) número de árboles huecos y arbustos.

Análisis de datos. Para la determinación de la estructura de la comunidad de anfibios y reptiles se compilaron los datos de todos los muestreos realizados obteniéndose la riqueza y el listado de las especies registradas. Se determinó el número total de individuos registrados por especie (abundancia relativa) y con ello se elaboró una gráfica rango-abundancia. Para determinar la diversidad de cada sitio, se utilizó el índice de Shannon-Wiener y de equitatividad (Magurran 1988). Para la determinación de los patrones espaciales de la comunidad, se combinaron los datos de los muestreos de ambas temporadas de trabajo para comparar las tendencias generales registradas en el BTC y la VA. Se calcularon los índices de diversidad y de similitud de especies mediante el índice de Jaccard (Magurran 1988, Brown \& Lomolino 1998) para cada uno de los sitios y estaciones de muestro. Se utilizaron análisis de varianza (ANOVA) para analizar las tendencias espaciales (tipo de vegetación, sitios y estaciones de muestreo) y temporales (temporadas de muestreo) en la riqueza, diversidad, equitatividad y abundancia dentro y entre los grupos taxonómicos (Pearman et al. 1995, Homyack \& Giuliano 2002). Además, se elaboraron dendrogramas a partir de los índices de similitud de especies por tipo de vegetación y estación de muestreo Se calcularon las curvas de acumulación y el número máximo de especies que se podrían encontrar en los tres sitios de muestreo utilizando distintos modelos predictivos por medio del programa EstimateS 5.0.1 (Colwell 1997). Así, se emplearon distintos estimadores para las curvas de acumulación de especies: Chao1, Chao2, Jack2 e ICE, que son los más recomendados (Colwell \& Coddington 1994). 
Otro análisis de las tendencias espacio-temporales de la comunidad incluyó el método no paramétrico de escalamiento multidimensional (NMS), el cual es un análisis multivariado de ordenación (McCune \& Mefford 1999) y para lo cual se empleo el programa NMS pc-ord (ver. 4.01). Se utilizaron dos enfoques, uno desde la perspectiva de los sitios y estaciones de muestreo, y otra desde la perspectiva de las especies. Así, primero se determinó las diferencias estacionales de la riqueza y de la composición de especies entre sitios y estaciones de muestreos, por lo que este análisis ordenó espacialmente dentro de la gráfica a los sitios y estaciones de muestreo de acuerdo a la presencia y ausencia estacional de las especies. Posteriormente, se tomó en cuenta la estación de muestreo por sitio y en cada temporada de muestreo para cada especie (presencia-ausencia) por lo que, en la gráfica resultante se presenta el ordenamiento espacial entre las especies de acuerdo a los sitios y estaciones de muestreo.

Para determinar el efecto de la estructura de la vegetación con los patrones espacio-temporales de la estructura de la comunidad de reptiles y anfibios, se utilizaron regresiones múltiples con el programa JMP 3.2.2, teniendo a las características de la estructura de la vegetación como los factores independientes mientras que la diversidad, riqueza, equitatividad y abundancia como las respuestas o variables dependientes.

\section{RESULTADOS}

Base de datos. Se registró un total de 1123 especímenes pertenecientes a 34 especies de reptiles (25) y anfibios (nueve). Del total de individuos registrados, 188 (ó el 16.74\%) fueron anfibios mientras que 935 (ó el 83.26\%) correspondió a reptiles. De la muestra registrada, 503 ejemplares corresponden a la época de lluvias y 620 a la de secas, representando respectivamente a 24 especies y 22 especies (Cuadro 1). Por tipo de vegetación, del número de individuos registrados, 504 fueron localizados en la SBC y 619 en la VA.

La gráfica de acumulación de especies (Fig. 3) muestra el número aproximado de especies que se podrían encontrar en los tres sitios de muestreo durante ambas temporadas. Los estimadores permitieron calcular el número potencial de especies entre 33.8 y 38.8 , dichas curvas alcanzaron los puntos más altos de la asíntota desde el muestreo 53, mientras que la Sobs (riqueza observada) alcanzó su asíntota en el muestreo 71 con 34 registros.

Estructura de la comunidad de reptiles y anfibios. La riqueza de especies fue casi tres veces mayor en reptiles ( 25 especies) que en anfibios ( 9 especies). La composición de las 25 especies de reptiles incluyó a 15 lagartijas y 10 serpientes mientras que todas las especies de anfibios registradas fueron anuros (ranas y sapos: Fig. 4). El análisis general de la abundancia de especies mostró que existen pocas especies muy abundantes y muchas poco abundantes (Fig. 4). El 75\% de los 
Cuadro 1. Especies registradas por temporada de muestreo.

\begin{tabular}{|c|c|c|c|c|}
\hline Clase & Especie & Lluvias & Secas & Ambas \\
\hline Amphibia & Chaunus marinus & $\mathrm{X}$ & & \\
\hline Amphibia & Ollotis marmorea & $\mathrm{X}$ & & \\
\hline Amphibia & Ollotis mazatlanensis & $\mathrm{X}$ & $\mathrm{X}$ & $\mathrm{x}$ \\
\hline Amphibia & Craugastor hobartsmithi & $\mathrm{X}$ & & \\
\hline Amphibia & Syrrophus nitidus & $\mathrm{X}$ & & \\
\hline Amphibia & Craugastor occidentalis & $\mathrm{X}$ & & \\
\hline Amphibia & Gastrophryne usta & $\mathrm{X}$ & & \\
\hline Amphibia & Dendropsophus sartori & & $\mathrm{X}$ & \\
\hline Amphibia & Hypopachus variolosus & $\mathrm{X}$ & $\mathrm{X}$ & $\mathrm{x}$ \\
\hline Reptilia & Agkistrodon bilineatus & $\mathrm{X}$ & & \\
\hline Reptilia & Ameiva undulata & $\mathrm{X}$ & $\mathrm{X}$ & $\mathrm{x}$ \\
\hline Reptilia & Anolis nebulosus & $\mathrm{X}$ & $\mathrm{X}$ & $\mathrm{x}$ \\
\hline Reptilia & Aspidoscelis communis & $\mathrm{X}$ & $\mathrm{X}$ & $\mathrm{x}$ \\
\hline Reptilia & Aspidoscelis lineattissima & $\mathrm{X}$ & $\mathrm{X}$ & $\mathrm{x}$ \\
\hline Reptilia & Coleonyx elegans & $\mathrm{X}$ & $\mathrm{X}$ & $\mathrm{x}$ \\
\hline Reptilia & Conophis vittatus & & $\mathrm{X}$ & \\
\hline Reptilia & Ctenosaura pectinata & $\mathrm{X}$ & & \\
\hline Reptilia & Eumeces parvulus & & $\mathrm{X}$ & \\
\hline Reptilia & Heloderma horridum & $X$ & $\mathrm{X}$ & $\mathrm{X}$ \\
\hline Reptilia & Leptotyphlops humilis & & $\mathrm{X}$ & \\
\hline Reptilia & Mabuya brachyopoda & $\mathrm{X}$ & & \\
\hline Reptilia & Manolepis putnami & $\mathrm{X}$ & $X$ & $\mathrm{x}$ \\
\hline Reptilia & Oxybelis aeneus & & $\mathrm{X}$ & \\
\hline Reptilia & Phyllogactylus lanei & $\mathrm{X}$ & & \\
\hline Reptilia & Rhadinea hesperia & & $\mathrm{X}$ & \\
\hline Reptilia & Salvadora mexicana & $X$ & & \\
\hline Reptilia & Sceloporus horridus & & $\mathrm{X}$ & \\
\hline Reptilia & Sceloporus melanorhinus & $\mathrm{X}$ & $\mathrm{X}$ & $\mathrm{x}$ \\
\hline Reptilia & Sceloporus utiformis & $\mathrm{X}$ & $\mathrm{X}$ & $\mathrm{x}$ \\
\hline Reptilia & Senticolis triaspis & & $\mathrm{X}$ & \\
\hline Reptilia & Sphenomorphus assatus & $\mathrm{X}$ & $\mathrm{X}$ & $\mathrm{x}$ \\
\hline Reptilia & Tantilla bocourti & & $\mathrm{X}$ & \\
\hline Reptilia & Tantilla calamarina & & $\mathrm{X}$ & \\
\hline$\underline{\text { Reptilia }}$ & Urusaurus bicarinatus & $\mathrm{X}$ & & \\
\hline
\end{tabular}




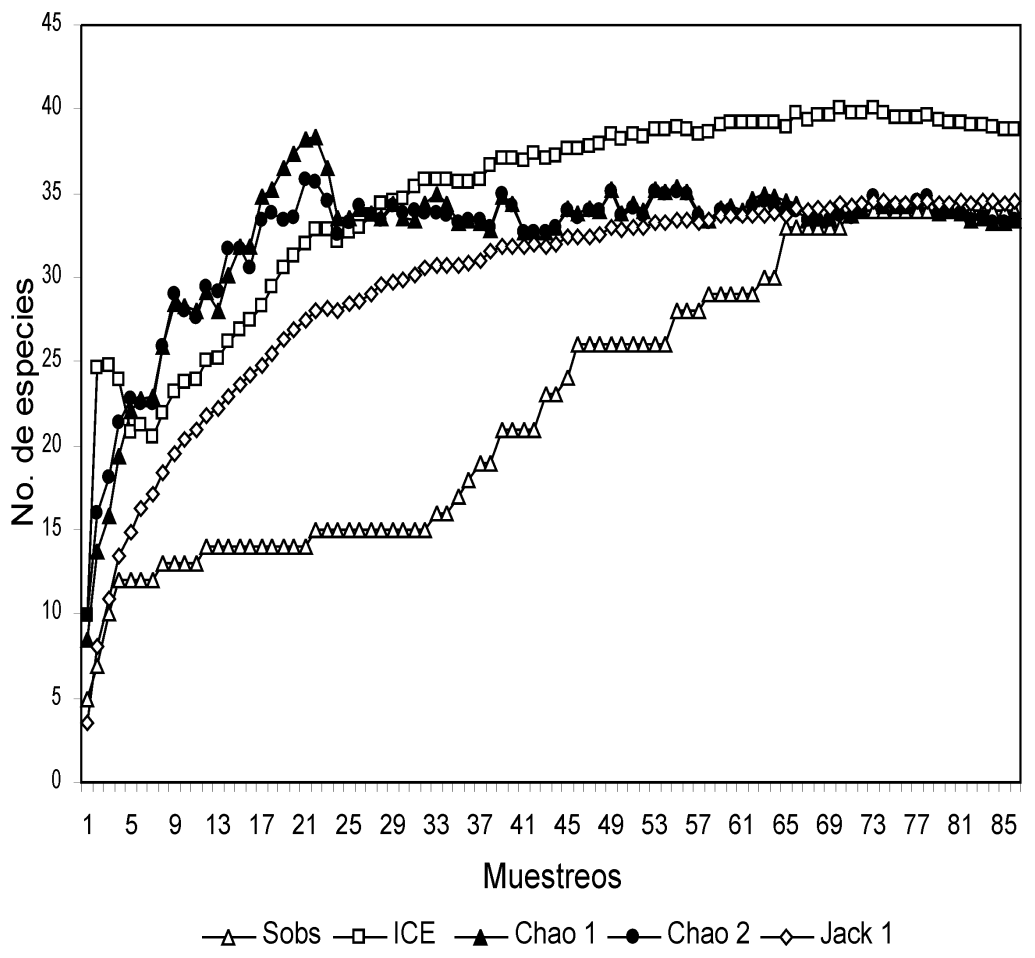

Figura 3. Curva de acumulación de especies después de 86 muestreos acumulados, combinando datos de los 3 sitios de estudio y de acuerdo a los modelos predictores de riqueza: Chao 1, Chao 2, Jack 1 e ICE, todos con 50 aleatorizaciones. Sobs = Riqueza observada $(S=34)$.

individuos registrados durante este estudio representan a tan sólo cuatro especies, tres de lagartijas y un sapo, que son en orden decreciente de abundancia, Aspidocelis lineattissima (27.6\% de la totalidad de los registros), Ameiva undulata (21.5\%), Sceloporus utiformis (18.3\%) y Ollotis marmorea (9\%). Para el resto de las especies, el número de registros fue mucho menor e incluso para algunas especies sólo se tuvo un registro en todo el estudio (Fig. 4). El valor del índice de diversidad, calculado con base en el total de los registros obtenidos fue casi dos veces mayor en reptiles $\left(\mathrm{H}^{\prime}=1.98\right)$ que en anfibios $\left(\mathrm{H}^{\prime}=1.07\right)$.

Tendencias espaciales. La riqueza, diversidad y equitatividad de especies no presentaron variaciones significativas entre los tres sitios de muestreo en anfibios $(\mathrm{gl}=2, \mathrm{~F}=1.66, \mathrm{P}>0.05, \mathrm{gl}=2, \mathrm{~F}=0.56, \mathrm{P}>0.05, \mathrm{y}$ g.l. $=2, \mathrm{~F}=0.05, \mathrm{P}>0.05$, respectivamente) ni en reptiles $(\mathrm{gl}=2, \mathrm{~F}=0.24, \mathrm{P}>0.05, \mathrm{gl}=2, \mathrm{~F}=0.33, \mathrm{P}>0.05$, y g.l. $=2, \mathrm{~F}=1.28, \mathrm{P}>0.05$, respectivamente) al combinar los datos ambas 


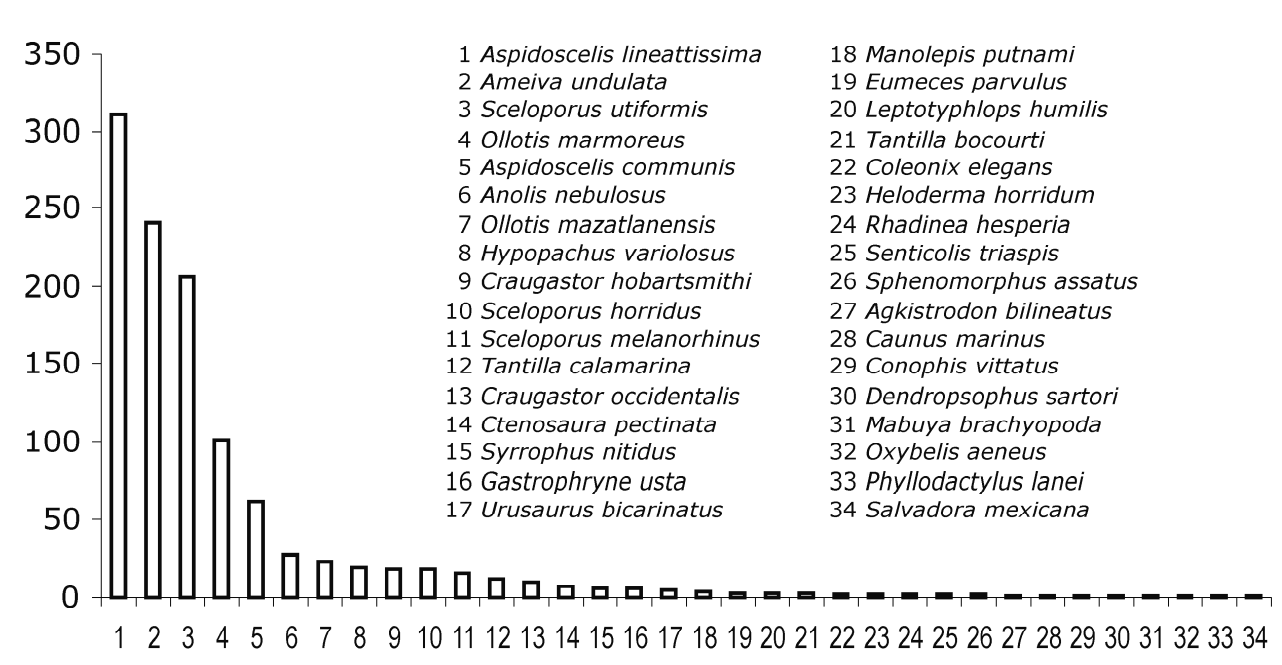

Especies

Figura 4. Abundancia relativa y composición de las especies registradas

temporadas de muestreo y tipos de vegetación. A continuación se describen las principales tendencias registradas: La mayor riqueza y diversidad de reptiles se registró en el Sitio 2 Calandria (18 especies, $\mathrm{H}^{`}=1.79$ ), seguido por el Sitio 1 Búho (17 especies, $\left.\mathrm{H}^{\prime}=1.77\right)$ y el Sitio 3 Hornitos $\left(14\right.$ especies, $\left.\mathrm{H}^{\prime}=1.69\right)$, mientras que la mayor equitatividad de los sitios se registró en el sitio 3 Hornitos $(\mathrm{E}=0.64)$ ligeramente por arriba de la registrada en los sitios 1 y $2(0.63$ y 0.61 , respectivamente). En el caso de los anfibios, la mayor riqueza y diversidad ocurrió en el Sitio 2 Calandria (ocho especies, $\mathrm{H}^{`}=1.62$ ), seguido por el Sitio 1 Búho (seis especies, $H^{`}=1.34$ ) y el Sitio 3 Hornitos (seis especies, $H^{`}=1.15$ ), entre tanto que la equitatividad fue similar en los sitios 1 y $2(\mathrm{E}=0.77)$, por arriba de la registrada en el sitios 3 Hornitos (0.64).

La riqueza de reptiles y anfibios no varió significativamente entre las 4 estaciones de muestreo $(\mathrm{gl}=3, \mathrm{~F}=0.12, \mathrm{P}>0.05 \mathrm{y} \mathrm{gl}=3, \mathrm{~F}=2.28, \mathrm{P}>0.05)$ al combinar $\operatorname{los}$ datos de todos los sitios de muestreo, a pesar de que la riqueza tanto de anfibios como de reptiles tendió a ser mayor hacia ambos extremos (E1 y E4) de la matriz VA-BTC (Cuadro 2).

Los índices de similitud de la herpetofauna calculados entre las estaciones de muestreo y combinando los datos de ambas temporadas de muestreo fueron muy bajos. La estación de muestreo ubicada en el centro de la VA (E1) registró valores inferiores de similitud con respecto al resto de las estaciones, lo que la separa claramente de las demás en términos de su composición de especies (Cuadro 3). 
García \&Cabrera: Estacionalidad y estructura de la vegetación en la comunidad herpetofaunistica

Cuadro 2. Riqueza de especies, de la herpetofauna, reptiles y anfibios por estación de muestreo, total (ambas) y por temporada (seca y lluvias) registrada durante este estudio.

\begin{tabular}{lccccccccc}
\hline & \multicolumn{3}{c}{ Herpetofauna } & \multicolumn{3}{c}{ Reptiles } & \multicolumn{2}{c}{ Anfibios } \\
\hline $\begin{array}{l}\text { Estación } \\
\text { de Muestreo }\end{array}$ & Secas & Lluvias & Ambas & Secas & Lluvias & Ambas & Secas & Lluvias & Ambas \\
\hline & & & & & & & & & \\
Estación 1 & 13 & 18 & 24 & 11 & 10 & 16 & 2 & 8 & 8 \\
Estación 2 & 13 & 16 & 22 & 12 & 9 & 15 & 0 & 7 & 7 \\
Estación 3 & 8 & 15 & 17 & 8 & 10 & 12 & 0 & 5 & 5 \\
Estación 4 & 12 & 18 & 24 & 11 & 11 & 16 & 1 & 7 & 8 \\
\hline
\end{tabular}

Cuadro 3. Índice de similitud de especies de la herpetofauna (Jaccard) para las distintas estaciones de muestreo durante las temporadas de muestreo.

\begin{tabular}{|c|c|c|c|c|c|c|c|c|c|c|c|c|}
\hline & \multicolumn{4}{|c|}{ Secas } & \multicolumn{4}{|c|}{ Lluvias } & \multicolumn{4}{|c|}{ Ambas } \\
\hline & E1 & E2 & E3 & E4 & E1 & E2 & E3 & E4 & E1 & E2 & E3 & E4 \\
\hline E1 & 1.00 & 0.37 & 0.32 & 0.32 & 1.00 & 0.61 & 0.57 & 0.63 & 1.00 & 0.37 & 0.31 & 0.32 \\
\hline E2 & & 1.00 & 0.61 & 0.47 & & 1.00 & 0.72 & 0.78 & & 1.00 & 0.62 & 0.47 \\
\hline E3 & & & 1.00 & 0.81 & & & 1.00 & 0.65 & & & 1.00 & 0.67 \\
\hline E4 & & & & 1.00 & & & & 1.00 & & & & 1.00 \\
\hline
\end{tabular}

Cuadro 4. Diversidad (H') por estaciones de muestreo, estación ambiental y grupo taxonómico.

\begin{tabular}{lcccccc}
\hline & \multicolumn{3}{c}{ Reptiles } & \multicolumn{3}{c}{ Anfibios } \\
\hline Estación & Secas & Lluvias & Ambas & Secas & Lluvias & Ambas \\
\hline Estación 1 & 1,10 & 0,77 & 0,93 & 0 & 1,12 & 0,56 \\
Estación 2 & 1,15 & 0,81 & 0,98 & 0 & 0,81 & 0,41 \\
Estación 3 & 1,25 & 0,86 & 1,05 & 0 & 0,64 & 0,32 \\
Estación 4 & 1,20 & 0,80 & 1,00 & 0 & 0,55 & 0,28 \\
\hline
\end{tabular}

No se encontraron diferencias significativas entre las estaciones de muestreo en la diversidad (Cuadro 4) de reptiles y anfibios $(\mathrm{gl}=3, \mathrm{~F}=0.84, \mathrm{P}>0.05 \mathrm{y} \mathrm{gl}=3, \mathrm{~F}=$ $0.85, \mathrm{P}>0.05)$. Tampoco se detectaron diferencias significativas en la equitatividad entre estaciones de muestreo en los anfibios ni en los reptiles $(\mathrm{gl}=3$, $\mathrm{F}=0.46, \mathrm{P}>0.05 \mathrm{y} \mathrm{gl}=3, \mathrm{~F}=1.52, \mathrm{P}>0.05$, respectivamente). Por último, la abundancia registrada entre estaciones de muestreo no fluctuó significativamente en reptiles $(\mathrm{gl}=3, \mathrm{~F}=0.46, \mathrm{P}>0.05)$ pero sí registró diferencias significativas en 
anfibios (g.l. = 3, F 5.22, p < 0.05), observándose una abundancia casi tres veces mayor en el centro (E1) de la VA y menor hacia las estaciones de muestreo ubicadas dentro de la BTC(E3 y E4: Cuadro 5).

Cuadro 5. Abundancia de individuos registrada por estaciones de muestreo y temporadas de muestreo para cada grupo taxonómico.

\begin{tabular}{lcccccccccccc}
\hline & \multicolumn{3}{c}{ Secas } & \multicolumn{1}{c}{ Lluvias } & \multicolumn{1}{c}{ Ambas } \\
\hline Taxa & E1 & E2 & E3 & E4 & E1 & E2 & E3 & E4 & E1 & E2 & E3 & E4 \\
\hline & & & & & & & & & & & & \\
Anfibios & 3 & 0 & 0 & 1 & 92 & 37 & 26 & 29 & 95 & 37 & 26 & 30 \\
Reptiles & 158 & 149 & 148 & 161 & 111 & 69 & 79 & 60 & 269 & 218 & 227 & 221 \\
Herpetofauna & 161 & 149 & 148 & 162 & 203 & 106 & 105 & 89 & 364 & 255 & 253 & 251 \\
\hline
\end{tabular}

Tendencias temporales. Al comparar la riqueza total registrada por temporada de muestreo (secas y lluvias), combinando los datos de todos los sitios y estaciones de muestreo, ésta fue ligeramente mayor durante las lluvias (24 especies) que en la temporada seca (22 especies). Sin embargo, el promedio de la riqueza herpetofaunística por muestreo fue significativamente mayor durante la época de lluvias (5.5 especies) que en las secas (3.5) $(\mathrm{g} .1 .=1, \mathrm{~F}=12.07, P<0.05)$. Las fluctuaciones estacionales de la riqueza total registrada de reptiles y anfibios mostraron tendencias opuestas, observándose una mayor riqueza de reptiles durante la época seca (19 vs. 16) y mayor de anfibios durante las lluvias (ocho vs tres), estas variaciones temporales sólo fueron estadísticamente significativas para los anfibios (g.l. $=1, \mathrm{~F}=82.83, P<0.05)$.

El análisis temporal (secas vs lluvias) de la similitud de especies registró un recambio importante de especies, más marcado en anfibios que en reptiles, como lo sugieren los valores del índice de Jaccard ( 0.22 y 0.40 , respectivamente). De acuerdo al análisis de escalamiento multidimensional (MNS) (Fig. 5) que toma en cuenta la riqueza de especies en cada sitio y estación de muestreo a través de cada temporada de muestreo (lluvias y secas), existió una clara diferencia (evidenciada por la separación de los puntos) de la riqueza y composición de especies entre sitios y estaciones de muestreo, con excepción de las estaciones de muestreo E3 del sitio 1 Búho y de E2 del sitio 2 Calandria, ya que durante la época de sequía, estos sitios registraron una riqueza y composición de especies similar (ocupan el mismo sitio en la Fig. 5). Resulta evidente la clara separación en la distribución de los puntos en los sitios por temporada, lo que apoya lo sugerido anteriormente por los índices de Jaccard obtenidos, en el sentido de la existencia de un recambio importante de especies de la herpetofauna a través de las épocas del año, y confirmado a nivel de estaciones (Fig. 6). 


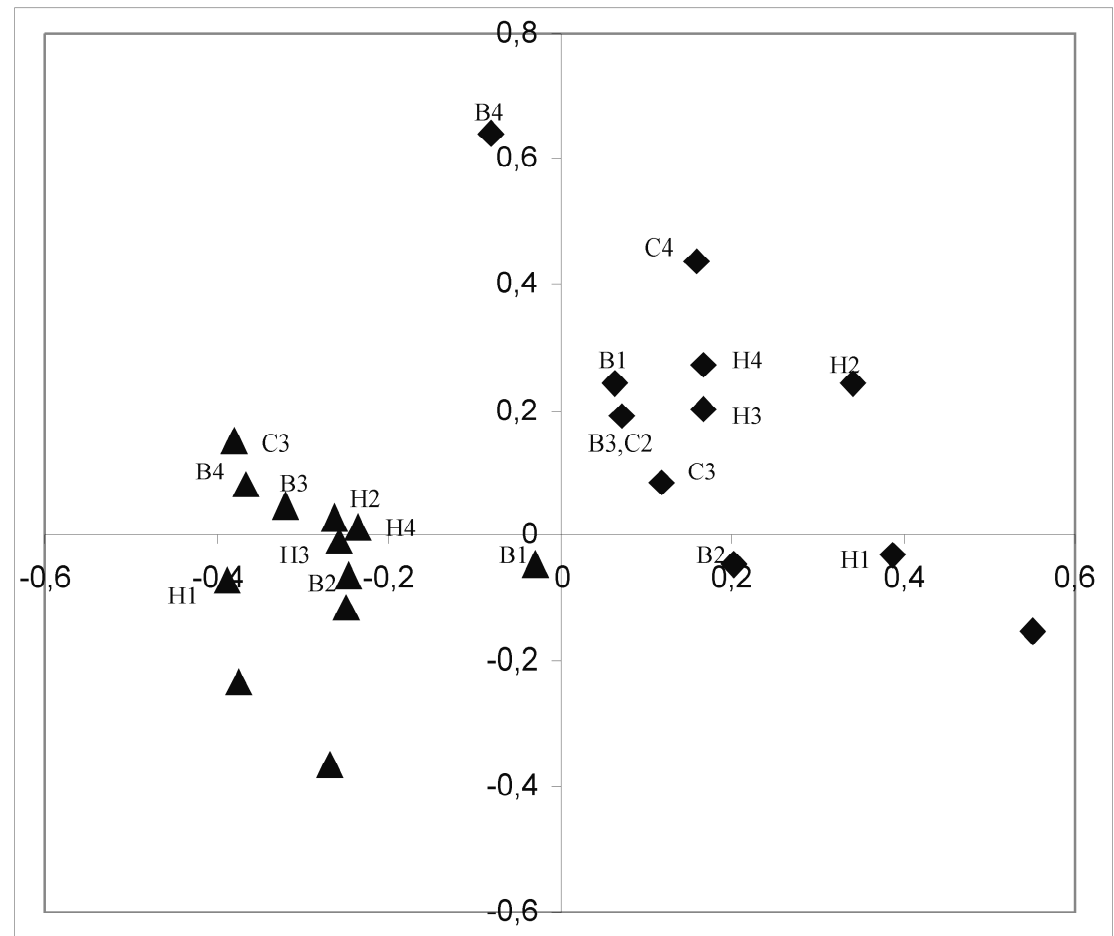

Figura 5. Análisis de escalamiento multidimensional de los sitios con base en su composición durante ambas temporadas ( secas y $\boldsymbol{\Delta}$ lluvias), (B Búho, C Calandria y H Hornitos) y (1 E1, 2 E2, 3 E3 Y 4 E4).

Con respecto a la abundancia, durante la época de secas se registró un mayor número de individuos (620) que en la época de lluvias (503). Las fluctuaciones estacionales en abundancia de reptiles y anfibios mostraron tendencias opuestas, registrándose una mayor abundancia de reptiles durante la secas (616 individuos vs 319 en lluvias) y de anfibios durante las lluvias (184 vs. 4 en secas). De esta manera, la abundancia de reptiles fue casi dos veces mayor en las secas que en lluvias, mientras que la de anfibios fue 46 veces mayor en las lluvias que en la época seca, siendo en ambos casos estas diferencias estadísticamente significativas (g.l $=1$, $\mathrm{F}=42.93, P<0.0001$ y g.l $=1, \mathrm{~F}=26.67, P<0.05$, respectivamente).

La diversidad de especies de la herpetofauna registró diferencias estacionales estadísticamente significativas, siendo mayor durante la época de lluvias que durante la época seca $\left(\mathrm{H}^{\prime}=2.3\right.$ y $\mathrm{H}^{\prime}=1.0$, respectivamente, g.1. $\left.=1, \mathrm{~F}=7.25, P<0.05\right)$, registrándose resultados similares con respecto a los valores de equitatividad obtenidos $(0.75$ y 0.39 , g.1. $=1, \mathrm{~F}=22.33, P<0.05)$. En el caso de los reptiles, si bien la diversidad y equitatividad fueron ligeramente mayores en la época de lluvias 


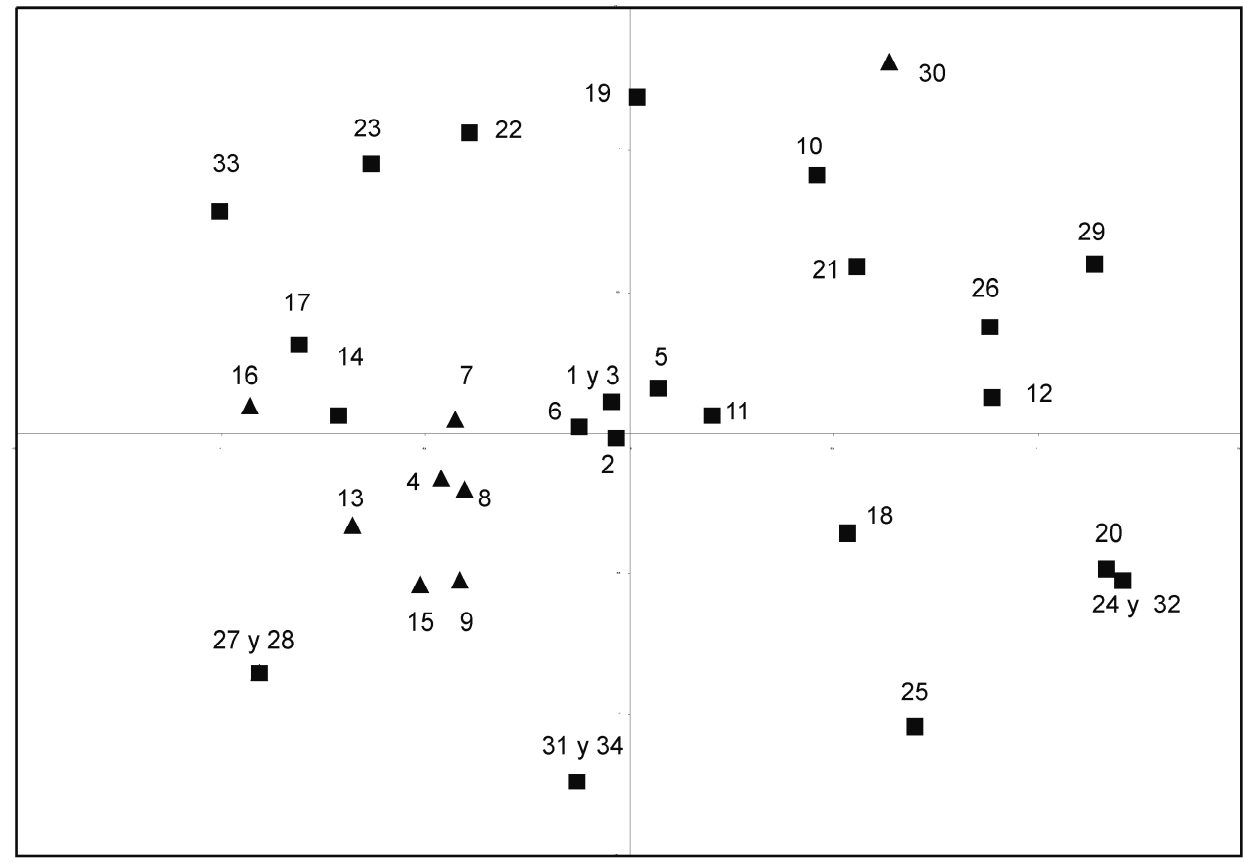

Figura 6. Análisis de escalamiento multidimensional de especies registradas en los sitios ( $\square$ reptiles y $\Delta$ anfibios).

que en la de secas $\left(\mathrm{H}^{\prime}=1.96\right.$ y $\mathrm{H}^{\prime}=2.0, \mathrm{y} \mathrm{E}=0.75 \mathrm{y} \mathrm{E}=0.70$, respectivamente), dichas diferencias no fueron estadísticamente significativas (diversidad $\mathrm{gl}=1, \mathrm{~F}=$ $0.09, \mathrm{P}>0.05$ y equitatividad $\mathrm{gl}=1, \mathrm{~F}=1.37, \mathrm{P}>0.05)$. En contraste, la mayor diversidad y más alta equitatividad de especies registrada para los anfibios durante la época de lluvias (33 y 9 veces mayor, respectivamente) con respecto a la época de secas $\left(\mathrm{H}^{\prime}=2.6 \mathrm{y} \mathrm{H}^{\prime}=0.08\right.$, y $\mathrm{E}=0.75 \mathrm{y} \mathrm{E}=0.08$, respectivamente $)$, fue en ambos casos estadísticamente significativas (g.l. $=1, \mathrm{~F}=7.95, P<0.05$ y g.l. $=1, \mathrm{~F}=35.36$, $P<0.05$, respectivamente).

Tendencias espacio-temporales. El análisis de la riqueza por temporada y tipos de vegetación registró ciertas variaciones estacionales, más evidentes en los anfibios que en los reptiles y en la herpetofauna en general (ambos grupos), siendo solamente estadísticamente significativas en el caso de los anfibios (g.l. $=1, \mathrm{~F}=112.43, P<$ $0.05)$. Así, el $66.6 \%$ de la riqueza de especies de anfibios registrada durante la temporada seca, fue encontrada en la VA (dos especies), mientras que durante la época de lluvias, la riqueza de especies de anfibios se distribuyó de forma uniforme a través de las estaciones de muestreo o tipos de vegetación, siendo ligeramente 
mayor en la VA (Cuadro 1). En el caso de los reptiles, la riqueza se distribuyó más uniformemente a través de las estaciones de muestreo o tipos de vegetación y con menores fluctuaciones temporales que en el caso de los anfibios (Cuadro 1).

La comparación estacional de la similitud de especies por tipos de vegetación para la herpetofauna en general (Cuadro 3), sugiere una mayor similitud de especies entre éstos durante la época de lluvias (0.66 en promedio) que durante la de secas $(0.48)$. Los dendrogramas elaborados a partir de los índices de similitud por tipo de vegetación y estación de muestreo, sugieren que, independientemente de la temporada de muestreo, la composición de especies presentes en el centro de la VA (E1) difiere del resto de la registrada en las otras tres estaciones de muestreo, mientras que durante la época seca estas diferencias son más evidentes dentro de la matriz VA-BTC (Fig. 7).

La abundancia de individuos por grupo, mostró variaciones estacionales por tipo de vegetación o estación de muestreo. En el caso de los reptiles, la abundancia fue mayor en la VA (E1) durante las lluvias y ligeramente mayor en el BTC (E4) durante la época de secas (Cuadro 5), mientras que en los anfibios, la abundancia fue mayor en la VA (E1) durante la época de lluvias y en las secas (Cuadro 4), resaltando la poca abundancia de individuos de este grupo durante la sequía y la tendencia a la disminución de la abundancia desde la VA hacia las estaciones más cercanas al BTC. La abundancia de anfibios en el centro (E1) de la VA fue hasta tres veces mayor, con respecto a otras estaciones de muestreo a lo largo de la matriz VA-BTC.

La diversidad de especies fluctuó estacionalmente, en especial en los anfibios para los cuales fue nula durante la época de secas, mientras que durante las lluvias alcanzó valores de $\mathrm{H}^{\prime}$ desde 0.55 hasta 1.12 en la estación de muestreo (E1), ubicada en el centro de la VA (Cuadro 4). En reptiles la diversidad fue mayor durante la época de secas en todas las estaciones de muestreo pero con valores más altos en las estaciones de muestreo dentro del BTC en ambas temporadas de muestreo (Cuadro 4).

El análisis multivariado de ordenación para las especies en las dimensiones dadas por los sitios y estaciones de muestreo para cada una de las temporadas muestra una clara tendencia de ocho especies de anfibios a agruparse en la gráfica probablemente por su mayor requerimiento de agua, mientras que en el centro de la gráfica se encuentran las especies de reptiles que se reportaron durante todo el muestreo y en todas las estaciones de monitoreo (Fig. 6). La especie de anfibio que está alejada del resto de los anfibios en esta gráfica, corresponde a Dendropsophus sartori que solo fue registrada una vez, en el BTC y en la época de secas. Los reptiles, a diferencia de los anfibios, se encuentran mejor distribuidos debido a que su disposición es más homogénea a través de la matriz VA-BTC y durante todo el muestreo (Fig. 6). 

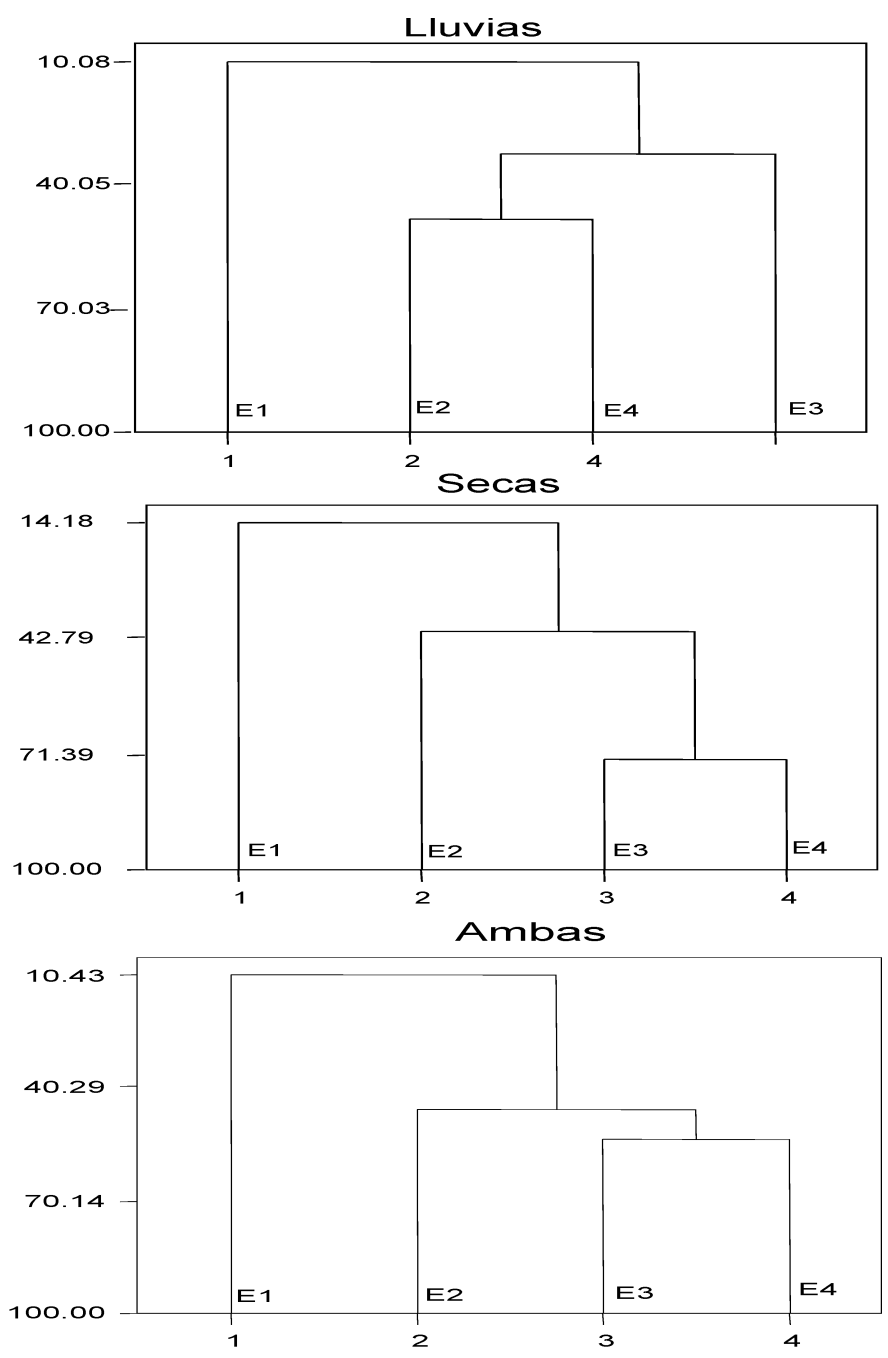

Figura 7. Dendograma de acuerdo al índice de similitud de especies de la herpetofauna (Jaccard) para las Estaciones de muestreo y por temporada de muestreo.

Efecto de la estructura de la vegetación. Al analizar los cambios en la riqueza, abundancia, diversidad y equitatividad en relación a diversos características de la estructura de la vegetación solo se encontraron relaciones significativas en la diversidad y equitatividad. Así, la diversidad de reptiles se relacionó significativamente con el número de ramas $\left(\mathrm{H}^{\prime}=2.47-0.0017\right.$ No. de Ramas: 
$\mathrm{P}=0.0414)$, mientras que la de anfibios con el número de ramas y el DAP $\left(\mathrm{H}^{\prime}=2.60\right.$ +0.1380 Pro Ramas $(P=0.0092)-0.1468$ DAP Pro $(0.0415)$, mientras que la equitatividad de anfibios se relacionó significativamente con varios aspectos de la estructura de la vegetación $(\mathrm{E}=52.16$ - 3.9240 DAP Pro $(1.0176)+0.1704$ No. de Bejucos (0.0077) + 0.3989 No. de Arbustos (0.0254) - 0.0575 No. de Ramas $(0.0165)+5.6039$ Pro Ramas $(0.0025)$.

\section{DISCUSIÓN}

En el presente estudio y mediante las tres técnicas de muestreo empleadas en todos los sitios y estaciones de muestreo, fue posible registrar en total 34 especies de anfibios y reptiles de las 87 especies documentadas en la región (García \& Ceballos 1994; Ramírez-Bautista 1994, Ramírez-Bautista \& García 2002, Ramírez-Bautista \& Moreno 2006), lo que corresponde casi el $46 \%$ de las 75 especies de las especies susceptibles de ser capturadas mediante las técnicas de muestreo utilizadas de acuerdo a un estudio previo realizado en la región (García 2003) en el cual registraron 54 especies en 60 meses de muestreo empleando 60 trampas de caída, mientras que en el presente se registraron 34 especies en tan solo 4 meses y empleando 72 trampas de caída pero con otras técnicas complementarias. Cabe señalar, que a diferencia del presente estudio, en el que se muestreo intensamente durante dos bimestres, durante los cinco años de muestreo realizados por García (2003), el muestreo si bien a más largo plazo, el trampeo solo fue durante 5 días consecutivos cada mes. Consideramos que en el trampeo intensivo como el realizado en el presente estudio, el desempeño de las técnicas de muestreo fue satisfactorio y los datos obtenidos son representativos de la comunidad bajo estudio. Lo anterior se refuerza por el hecho de que la riqueza registrada en este estudio fue muy similar al rango predicho por los estimadores potenciales de la riqueza y por las curvas de acumulación de especies obtenidas (Hofer \& Bersier 2001, Gardner et al. 2007). En el contexto de los estudios de inventario herpetofaunísticos de comunidades del BTC los resultados obtenidos en este estudio, comparados con otros a más largo plazo (e.g. García 2003, Gardner et al. 2007) sugieren que las técnicas empleadas pueden ser una herramienta adecuada a corto plazo o cuando los recursos financieros y humanos son limitados.

Como se esperaba, la diversidad y riqueza de los reptiles fue mayor que en los anfibios, lo que coincide con los resultados previos obtenidos en un estudio previo (García 2003) y que corresponde al hecho de que en la región, la riqueza de reptiles es mayor (68 especies) que la de anfibios (19; García \& Ceballos 1994; RamírezBautista 1994, Ramírez-Bautista \& García 2002, Ramírez-Bautista \& Moreno 2006). Al igual que en otras comunidades naturales, la comunidad herpetofaunística de Chamela está caracterizada por un número considerable de especies relativamente poco abundantes y una cantidad reducida de especies con abundancia relativa alta (Ricklefs 2001, Gardner et al. 2007). 
Los resultados de la estructura de la comunidad de reptiles y anfibios sugieren variaciones espaciales, especialmente entre los tipos de vegetación, BTC y VA. La ausencia de significancia estadística de las variaciones entre estaciones de muestreo a lo largo de la matriz VA-BTC, probablemente se deba a que la variación espacial es sutil entre las estaciones de muestreo más cercanas, y que solo son notorias entre las estaciones de muestreo a ambos extremos del sitio del muestreo ubicadas dentro de la VA y el BTC. Lo anterior fue apoyado por los valores de los índices de similitud de especies que mostraron que existen algunas diferencias a lo largo de la matriz VABTC donde las estaciones de muestreo ubicadas en la VA claramente se separan del resto en términos de su composición de especies. La mayor riqueza, abundancia relativa, diversidad y equitatividad de especies de reptiles y en especial la de anfibios registrada en VA, puede explicarse por la menor estacionalidad ambiental y mayor productividad y complejidad estructural de este tipo de vegetación en comparación con el BTC (Bullock et al. 1995, Lott et al. 1987). El efecto positivo de la complejidad estructural del hábitat y la productividad sobre la riqueza de especies se ha reportado ampliamente para diversos grupos de organismos y una amplia gama de ambientes (e.g. Bersier \& Meyer 1994, Pearman 1997, Tilman et al. 1997, Kaspari et al. 2000). En el caso especifico de la región de Chamela, trabajos previos han encontrado resultados similares al presente estudio, detectándose una mayor riqueza y abundancia de mamíferos en la VA que en el BTC, en especial durante la época de secas (Ceballos 1989, 1990, Mandujano \& Gallina 1996, Mandujano 1999, Nuñez \& Miller 2000, Valenzuela \& Ceballos 2000): sin embargo, no fue el caso de las avifauna, probablemente debido a la gran movilidad de las especies, cambios estacionales en la composición de las especies por migración, y debido a su conducta reproductiva (Ornelas et al. 1993). Así mismo, en otros ambientes áridos y estacionales, tales como los desiertos, durante la época de secas, la distribución de las especies de reptiles y anfibios se concentra en los arroyos o ecosistemas con menor estacionalidad ambiental, mayor disponibilidad de recursos y mayor complejidad estructural (Baker 1990, McComb et al. 1993a \& b; Degenhardt et al. 1996, Gómez \& Anthony 1996).

El hecho de que existan algunas especies de anfibios concentradas en la VA, hace que este tipo de vegetación pueda ser considerada como un refugio para el grupo cuando las condiciones de sequía en la región son considerables. Así, al llegar las lluvias, y como lo sugieren los dendrogramas elaborados a partir de los índices de similitud, las especies de anfibios se distribuyen homogéneamente en ambos tipos de vegetación, una vez que las condiciones de microclima y alimento en éstas son más similares que durante la época de secas. La importancia de este tipo de vegetación en el mantenimiento de la riqueza y diversidad herpetofaunística de la región como ha sido sugerido anteriormente a nivel de comunidad (García 2003) así como para las comunidades de mamíferos (Ceballos 1989, 1990, Mandujano \& Gallina 1996, 
Mandujano 1999, Nuñez \& Miller 2000, Valenzuela \& Ceballos 2000) y para los vertebrados en general (Ceballos 1995; Noguera et al. 2002).

Los resultados sugieren un mayor efecto de la estacionalidad ambiental sobre los anfibios que en reptiles a nivel de comunidad. Se encontraron fluctuaciones temporales en la estructura de la comunidad (riqueza, abundancia, diversidad y equitatividad), con cambios más marcados para los anfibios que en reptiles, existiendo un recambio estacional de especies, en especial de anfibios, como es sugerido en estudios previos en diversos hábitats (e.g. Abe 1995, Androne et al. 2001, García 2003). Lo anterior es notorio al observar que las tendencias temporales registradas en reptiles y anfibios fueron en cierta manera opuesta, la mayor riqueza y abundancia de reptiles durante las secas y la de anfibios durante las lluvias. Los resultados del análisis de escalamiento multidimensional mostraron una evidente separación de los sitios de acuerdo a la temporada de muestreo, implicando que la distribución espacial de las especies fue más homogénea durante la época de lluvias.

Las diferencias en las tendencias temporales en la riqueza, abundancia y diversidad de los reptiles y anfibios en la región de Chamela y otros ambientes estacionales pueden deberse, en parte, a la contrastante fisiología de estos grupos haciendo que la ocurrencia de sus especies, sus niveles y tipos de actividad difiera por los efectos de la estacionalidad ambiental (Baker 1990, McComb et al. 1993a \& b, Gómez \& Anthony 1996, Androne et al. 2001). Así, en esta comunidad existe baja presencia general de anfibios durante la época de secas y una presencia más o menos constante de reptiles a lo largo del año, el $22 \%$ de las especies de anfibios se registraron en ambas épocas contrastando con el $40 \%$ registrado en reptiles. La región de Chamela se caracteriza por una marcada estacionalidad en la disponibilidad de agua y por un ambiente con fluctuaciones estacionales en la temperatura relativamente menos marcadas (Bullock 1986, Noguera et al. 2002). Así, al ser los reptiles en general, más susceptibles a los cambios en la temperatura y los anfibios hacia los cambios en la disponibilidad de agua (Pough et al. 1998), la ocurrencia y actividad de los anfibios de Chamela responden a la estacionalidad de las lluvias, mientras que los reptiles, al registrarse relativamente poca variación estacional en la temperatura máxima (al medio día: Bullock 1986) pueden ocurrir durante prácticamente todo el año (García 2003). El efecto de la estacionalidad en las lluvias sobre las fluctuaciones en las comunidades de anfibios ha sido documentado en otras áreas tropicales, en especial en aquellas regiones marcadamente estacionales (Toft \& Duellman 1979, Duellman 1995, Duellman \& Thomas 1996). En el caso de los reptiles, como se mencionó anteriormente el efecto de la estacionalidad a nivel de comunidad (diversidad y riqueza) es menor que en anfibios, sin embargo, a nivel de especie e individuos, la estacionalidad, a través de los cambios en la disponibilidad de recursos, precipitación, y cambios en la estructura de la vegetación entre otros, tiene un efecto muy importante en los niveles de actividad y reproducción (Beck \& 
Lowe 1991, Lister \& García 1992, García \& Ceballos 1994, Ramírez-Bautista 2004; 1994).

Existieron diferencias estructurales de la vegetación a lo largo de la matriz VABTC, registrándose una mayor complejidad hacia las estaciones de muestreo ubicadas en o cerca de la VA concordando con lo reportado previamente en la región (Lott et al. 1987). La estructura de la vegetación tuvo diversos efectos en 1 reptiles y anfibios. Es interesante que cinco de las nueve características de la estructura de la vegetación incluidas en los modelos de regresiones múltiples resultasen responder (positiva o negativamente) a los factores analizados, los cuales afectaron la presencia de estos grupos taxonómicos. Aunque se sabe que la estructura de la vegetación no define los atributos de la comunidad herpetofaunística por completo, debido principalmente a que algunos de los organismos que componen a esta comunidad tienen hábitos fosoriales, terrestres o acuáticos y no arborícolas (García \& Ceballos 1994), se considera que al menos para las especies registradas en este estudio, los anfibios (diversidad y equitatividad) respondieron en mayor grado a los cambios en la estructura de la vegetación que los reptiles (diversidad), especialmente hacia DAP pro, Pro Ramas, No. de ramas, No. de arbustos y No. bejucos. La mayor complejidad estructural registrada en la VA dentro de la matriz VA-BTC, junto con la respuesta a los diversos factores de la estructura por parte de la comunidad de reptiles y anfibios, probablemente sea en parte la causa de la mayor riqueza, diversidad y abundancia de especies registradas en general por la VA, en contraste con el BTC. Otros factores como la mayor productividad y mayor disponibilidad de agua (por lo menos en el subsuelo y en la forma de humedad relativa) registrada en la VA, en comparación con el BTC, se han sugerido como la causa de la mayor diversidad de mamíferos e insectos en este tipo de vegetación (Ceballos 1989, 1991, Noguera et al. 2002), se requiere sin embargo, medir la respuesta de la herpetofauna a dichos factores para corroborar esta idea.

Varios trabajos se han enfocado a dilucidar las asociaciones especies-vegetación en vertebrados, especialmente en aves y mamíferos, aunque en menor grado en reptiles y anfibios. Entre estos últimos, las asociaciones con la vegetación han demostrado ser particularmente importantes (Coulson 1993, Williams et al. 1997, Sanders \& Edge 1998). De igual manera, la importancia de la estructura del hábitat en reptiles y anfibios en varios ambientes y a diferentes escalas ha sido determinada (e.g. Pianka 1967, Vallan 2002, García \& Whalen 2003). Este tipo de estudios, en los que se determinan los mecanismos que promueven y mantienen la diversidad a nivel de comunidad, generan información de gran importancia de tipo ecológico con implicaciones considerables en conservación (Brown 1995).

En conclusión, la estructura de la comunidad de reptiles y anfibios, si muestra tendencias espaciales y temporales, relacionadas con las fluctuaciones estacionales medioambientales registradas en el BTC y la VA de Chamela, siendo los anfibios el 
grupo más susceptible a las fluctuaciones medioambientales, al ser el grupo limitado por la extensión y disponibilidad de agua, su actividad y diversidad se reduce durante la época de secas, cuando la VA se puede utilizar como refugio para ciertas especies. Las tendencias menos estacionales en reptiles se pueden atribuir a la relativa constancia de temperatura y a las adaptaciones conductuales y fisiológicas de este grupo a la marcada estacionalidad del BTC. No existe un gradiente como tal de diversidad dentro de la matriz VA-BTC, pero si marcadas diferencias entre tipos de vegetación con la VA registrando los valores máximos de los atributos de la comunidad, durante todo el estudio.

Agradecimientos. Deseamos reconocer el apoyo recibido por el Dr. Ricardo Ayala Barajas, anterior jefe de la Estación de Biología Chamela, a través de la beca recibida para cubrir la estancia de A. Cabrera Reyes durante el desarrollo del presente trabajo. Agradecemos las revisiones y comentarios recibidos a las versiones preliminares de este trabajo por parte de David Valenzuela, Néstor Mariano y Jorge Morales, así como a los tres revisores anónimos que revisaron el manuscrito haciendo comentarios que mejoraron considerablemente su contenido.

\section{LITERATURA CITADA}

Abe, A. S. 1995. Estivation in south-american amphibians and reptiles. Braz. J. Med. Biol. Res. 28:1241-1247.

Almond, W. D. 1991. A plot study of forest floor litter frogs, Central Amazon, Brazil. J. Trop. Ecol. 7:503-522.

Androne, F., Vences, M. \& J. E. Randrianirina. 2001. Patterns of amphibian and reptile diversity at Berara Forest (Sahamalaza Peninsula), NW Madagascar. Boll. Zool. 68:235 -241.

Baker, W. L. 1990. Species richness of Colorado riparian vegetation. J. Veg. Sci.1: 119-124.

Beck, D.D. \& C. H. Lowe. 1991. Ecology of the beaded lizard Heloderma horridum in a tropical dry forest in Jalisco, Mexico. J. Herpetol. 25:395-406.

Beebee, T. J. 2002. Amphibian phenology and climate change. Conserv. Biol. 16:1454-1455.

Bersier, L.F. \& D. R. Meyer. 1994. Bird assemblages in mosaic forests: the relative importance of vegetation structure and floristic composition along the successional gradient. Acta Oecol. Int. J. Ecol. 15:163-174.

Brown, J. H. 1995. Macroecology. The University of Chicago Press.Brown, J. H. \& M. V. Lomolino. 1998. Biogeography, 2nd edition. Sinuaer Associates. Massachusetts, USA.

Bullock, S. 1986. Climate of Chamela, Jalisco, and trends in the south coastal region of Mexico. Arch. Met. Geaph. Biocl., Ser. B 36:297-316.

Bullock, S. Mooney, H. y E. Medina, editors. 1995. Seasonal dry forests. Cambridge University Press, Cambridge, United Kindom.

Ceballos, G. 1989. Population and community ecology of small mammals in a tropical deciduous forest in Western Mexico. Tesis doctoral. University of Arizona, Tucson, USA.

1990. Comparative natural history of small mammals from tropical forests in Western Mexico. J. of Mammal. 71:263-266.

1995. Vertebrate diversity, ecology, and conservation in neotropical dry forest. Pp. 195-219. In:

S. Bullock, H. Mooney and E. Medina (Eds). Seasonal dry forests. Cambridge University Press, Cambridge, United Kingdom. 
Ceballos, G. Szekely, A. García, A. Rodríguez, P. \& F. Noguera. 1999. Plan de manejo de la Reserva de la Biosfera Chamela-Cuixmala. SEMARNAP, Mexico D.F.

Colwell, R. K. 1997. EstimateS 7.5.0. Statistica Estimation of Species Richness and Shared Species for Simples. http://viceroy.eeb.uconn.edu/estimates

Colwell, R.K. \& J. A. Coddington. 1994. Estimating terrestrial biodiversity thought extrapolation. Philos. Trans. R. Soc. Lond. B. Biol. Sci. (Serie b) 345:101-118.

Coulson, G. 1993. Use of heterogeneous habitat by the western gray kangaroo, Macropus fuliginosus. Wildlife Res. 20:137-149.

Crump, M. L. \& N. J. Scott, Jr. 1994. Visual encounter surveys. Pp. 84-92. In: W. R. Heyer, M. A. Donelly, R. W. McDiarmid, L.-A. Hayek, \& M. S. Foster (Eds), Measuring and monitoring biological diversity: standard methods for amphibians. Smithsonian Institution Press, Washington, D.C.

Degenhardt, W. G., C. W. Painter \& A. H. Price. 1996. Amphibians and reptiles of New Mexico. University of New Mexico Press. USA.

Duellman, W. E. 1995. Temporal fluctuations in abundances of anuran amphibians in a seasonal Amazonian rain-forest. J. Herpetol. 29:13-21.

Duellman, W. E. \& R. Thomas. 1996. Anuran amphibians from seasonally dry forest in southeastern Peru and comparisons of the anuran among sites in the upper Amazon Basin. Occas. Pap. Mus. Nat. Hist. Univ. Kansas 180:1-34.

Fauth, J. E. Crother, B. I. \& J. B. Slowinski. 1989. Elevational Patterns of Species Richness, Evenness, and Abundance of the Costa Rican Leaf-Litter Herpetofauna. Biotropica, 21:178-185.

García, A. 2003. Biogeography, ecology and conservation of tropical dry forest herpetofauna in Western Mexico. Tesis doctoral. University of New Mexico. Albuquerque, NM., USA.

2006. Using Ecological Niche Modeling to Identify Diversity Hotspots of the Herpetofauna of Pacific Lowlands and Adjacent Interior Valleys of Mexico. Biol. Conserv. 130:25-46.

García, A. \& G. Ceballos. 1994. Guía de campo de los reptiles y anfibios de la costa de Jalisco, México. Fundación Ecológica de Cuixmala - Instituto de Biología, UNAM, Mexico, D.F.

García, A. \& D. Whalen. 2003. Lizard community structure along an inland desert-intertidal transition zone on the coast of Sonora, Mexico. J. Herpetol. 37:378-382.

Gardner, T. A. Fitzherbert, E. B. Drewes, R. C. Howell, K. M. \& T. Caro. 2007. Spatial and temporal patterns of abundance and diversity of an east African leaf litter amphibian fauna. Biotropica 39:105-113.

Gomez, D. M. \& R. G. Anthony. 1996. Amphibian and reptile abundance in riparian and upslope areas of 5 forest types in Western Oregon. Northwest Sci.70:109-119.

Heyer, W. R. \& K. A. Berven. 1973. Species diversity of herpetofaunal sample from similar microhabitats at two tropical sites. Ecology 54:642-645.

Heyer, W. R. Donnelly, M. A. McDiarmid, R. W. Hayek, L. C. \& M. S. Foster (Eds). 1994. Measuring and monitoring biological diversity: standard methods for amphibians. Smithsonian Institution Press, Washington, D.C.

Heinen, J. T. 1992. Comparisons of the litter herpetofauna in a abandoned cacao plantations and a primary rain forest in Costa Rica: some implications for faunal restoration. Biotropica 24:431-439.

Hofer, U. \& L. F. Bersier. 2001. Herpetofaunal Diversity and Abundance in Tropical Upland Forest of Cameroon and Panama. Biotropica, 33:142-152.

Hofer, U. Bersier, L. F. \& D. Borcard. 1999. Spatial organization of a herpetofauna on an elevational gradient revealed by null model tests. Ecology, 80:976-988.

Homyack, J. D. \& W. M. Giuliano. 2002. Effect of streambank fencing on herpetofauna in pasture stream zones. Wildl. Soc. Bull. 30: 361-369. 
Kaspari, M., O. S. Donnell y J. R. Kercher. 2000. Energy ; density ; and constraints to species richness: Ant assemblages along a productivity gradient. Am. Nat. 155:280-293

Leighton, M. \& D. R. Leighton. 1983. Vertebrate responses to fruiting seasonality within a Bornean rain forest. Pp. 181-195. In: Sutton, S.L., Withmore, T. C., and A. C. Chadwick (eds). Tropical rain forest: ecology and management. Special Publication, No. 2. British Ecological Society. Blackwell Scientific Publications. Oxford.

Lister, B. 1980. Resource variation and the structure of British bird communities. Proc. Nat. Acad. Sci. USA 77:4185-4187.

Lister, B. \& A. García. 1992. Seasonality, predation and, behavior of a mainland anole. J. Anim. Ecol. 61:717-733.

Lott, E.J. Bullock, S. H. \& A. Solis-Magallanes. 1987. Florisitic diversity and structure of upland and arroyo forests of coastal Jalisco. Biotropica 19:228-235.

MacArthur, R. H. \& J. W. MacArthur. 1961. On bird species diversity. Ecology 42:594-598.

McCune, B. \& M.J. Mefford. 1999. Non-metric Multidimensional Scaling (NMS). pc-ord ver. 4.01

Magurran, A. E. 1998. Ecological Diversity and Its Meashurment, Princeton University Press, Princeton, New Jersey, USA.

Mandujano, S. 1999. Variation in herd size of collared peccaries in a Mexican tropical forest. Southwestern Nat. 44:199-204.

Mandujano, S. \& S. Gallina. 1995. Disponibilidad de agua para el venado cola blanca en un bosque tropical caducifolio de México. Vida Silvestre Neotrop. 4:107-118.

1996. Size and composition of white-tailed deer groups in a tropical dry forest in Mexico. Ethol. Ecol. Evol. 8:255-263.

Martínez-Yrízar, A. J. M. Maass, L. A. Pérez-Jiménez \& J. Sarukhán. 1996. Net primary productivity of a tropical deciduous forest ecosystem in western Mexico. J. Trop. Ecol. 12:169-175.

McComb, W. C. Chambers, C. L. \& M. Newton. 1993a. Small mammal and Amphibian communities and habitat associations in red alder stands, central Oregon Coast Range. Northwest Sci. 67:181-208.

McComb, W. C. McGarigal, K. \& R. G. Anthony. 1993b. Small mammal and amphibian abundance in streamside and upslope habitats of mature Douglas-fir stands, western Oregon. Northwest Sci. 67: 7-15.

Metts, B. S. Lanham, J. D. \& K. R. Russell. 2001. Evaluation of herpetofaunal communities on upland streams and beaver-impounded streams in the Upper Piedmont of South Carolina. Amer. Midl. Natur. 145:54-65.

Murphy, P. G. \& A. E. Lugo. 1986. Ecology of tropical dry forest. Annu. Rev. Ecol. Syst. 17:67-88.

Noguera, F. Vega-Rivera, J. H. García-Aldrete, A. N. \& M. Quesada-Avendaño (Eds). 2002. Historia Natural de Chamela Instituto de Biologia, UNAM. México D.F. México.

Nuñez, R. \& B. Millar. 2000. Food habits of jaguars and pumas in Jalisco, Mexico. J. Zool. 252:373379.

Ornelas, J. M. Arizmendi, M. del C. Marquez-Valdelamar, L. \& M. de L. Navarijo. 1993. Variability profiles for line transect bird censuses in a tropical dry forest in Mexico. The Condor 95:422441.

Pearman, P. B. 1997. Correlates of amphibian diversity in an altered landscape of Amazonian Ecuador. Conserv. Biol. 11:1211-1225.

Pearman, P. B. Velasco, A. M. \& A. López. 1995. Tropical amphibian monitoring: a comparison of methods for detecting inter-site variation in species' composition. Herpetologica 51:325-337.

Pianka, E. 1967. On lizard species diversity: North American flatland deserts. Ecology 48:333-351.

Pough, F. H. Andrews, R. H. Cadle, J. E. Crump, M. L. Savitzky, A. H. \& K. D. Well. 1998. Herpetology. Prentice Hall. New Jersey. 
Potts, M. D. 2003. Drought in a Bornean everwet rain forest. J. Ecol. 91:467-474.

Ramírez-Bautista, A. 1994. Manual y claves ilustradas de los anfibios y reptiles de la región de Chamela, Jalisco, México. Cuadernos del Instituto de Biología No. 23. Universidad Nacional Autónoma de México, D.F.

Ramírez-Bautista, A. 1995. Demografía y Reproducción de la lagartija arborícola Anolis nebulosus de la región Chamela Jalisco. Tesis de Doctorado. Facultad de Ciencias, Universidad Nacional Autónoma de México, D.F.

2004. Diversidad de estrategias reproductivas en un ensamble de lagartijas de una región tropical estacional de las costas del Pacífico de México. Bol. Soc. Herpetol. Mex. 12(1):7-16.

Ramírez-Bautista, A. \& A. García. 2002. Diversidad de la herpetofauna de la región de Chamela. Pp. 251-263. In: F. Noguera, J. H. Vega-Rivera, A. N. García-Aldrete \& M. Quesada-Avendaño (Eds). Historia Natural de Chamela, Instituto de Biología, UNAM. México D.F. México.

Ramírez-Bautista, A. \& C. E. Moreno. 2006. Análisis comparativo de la herpetofauna de cuatro regiones geográficas de México. In: A. Ramírez-Bautista, L. Canseco-Márquez \& F. MendozaQuijano (Eds.) Inventarios Herpetofaunísticos de México: avances en el conocimiento de su biodiversidad. Publicación Especial, Sociedad Herpetológica Mexicana, A.C. pp. 74-98.

Ricklefs, R. E. 2001. The Economy of Nature. Fifth Edition. Freeman Bookshelf.Szaro, R. C. \& S. C. Belfit. 1986. Herpetofaunal use of a desert riparian island and its adjacent scrub habitat. $J$ Wildl Manage. 50:752-761.

Scott, N. J. 1976. Abundance and diversity of the herpetofaunas of tropical forest litter. Biotropica. 8:41-58.

Sanders, T. A. \& W. D. Edge. 1998. Breeding bird community composition in relation to riparian vegetation structure in the western United States. J. Wildl. Manage. 62:461-473.

Tews, J. Brose, U. Grimm, V. Tielbörger, K. Whichman, M. C. Schwager, M. \& F. Jeltsch. 2004. Animal species diversity driven by habitat heterogeneity: the importance of keystone structures. $J$. Biogeogra. 31:79-82.

Toft, C.A. \& W.E. Duellman. 1979. Anurans of the lower Rio Llullapichis, Amazonian Peru: A preliminary analysis of community structure. Herpetologica 35:71-77.

Tilman, D., C. L. Lehman, C. L. y K. T. Thomson. 1997. Plant diversity and ecosystem productivity: theoretical considerations. Proc. Nat. Acad. Sci. USA 94:857-1861

Trejo-Vázquez, I. 1998. Distribución y diversidad de selvas bajas de México: relaciones con el clima y el suelo, Tesis de Doctorado en Ciencias (Biologia), Facultad de Ciencias, UNAM, México.

Trejo-Vázquez, I. \& R. Dirzo. 2000. Deforestation of seasonally dry forest: a national and local analysis in Mexico. Biol. Conserv. 94:133-142.

Vallant, D. 2002. Effects of anthropogenic environmental changes on amphibian diversity in the rain forests of eastern Madagascar. J. Trop. Ecol. 18:725-742.

Valenzuela, D. \& G. Ceballos. 2000. Habitat selection, home range, and activity of the white-nosed coati (Nasua narica) in a Mexican tropical dry forest. J. Mammal. 81:810-819.

Villers-Ruiz, L. \& I. Trejo-Vázquez. 1997. Assessment of the vulnerability of forest ecosystems to climate change in Mexico. Clim. Res. 9:87-93.

Williams, B. K. Mahabir, S. Schlagel, J. \& D. E. Capen. 1997. Patterns in wildlife-vegetation association matrices. J. Environ. Manage. 51:1-13.

Wolda, H. 1988. Seasonality and the community. Pp. 69-95. In: J. H. Gee \& P. S. Giller (Eds). The Organization of the communities. Blackwell Scientific Publications, Oxford. 
